\title{
Phenotypes of craniofrontonasal syndrome in patients with a pathogenic mutation in EFNB1
}

\author{
MEP van den Elzen ${ }^{1}$, SRF Twigg ${ }^{2}$, JAC Goos ${ }^{1}$, AJM Hoogeboom ${ }^{3}$, AMW van den Ouweland ${ }^{3}$, \\ AOM Wilkie $^{2}$ and IMJ Mathijssen ${ }^{1}$
}

Craniofrontonasal syndrome (CFNS) is an X-linked developmental malformation, caused by mutations in the EFNB1 gene, which have only been described since 2004. A genotype-phenotype correlation seems not to be present. As it is of major importance to adequately counsel patients with EFNB1 mutations and their parents, and to improve diagnosis of new patients, more information about the phenotypic features is needed. This study included 23 patients ( 2 male, 21 female) with confirmed EFNB1 mutations. All patients underwent a thorough physical examination and photographs were taken. If available, radiological images were also consulted. Hypertelorism, longitudinal ridging and/or splitting of nails, a (mild) webbed neck and a clinodactyly of one or more toes were the only consistent features observed in all patients. Frequently observed phenotypic features were bifid tip of the nose (91\%), columellar indentation (91\%) and low implantation of breasts (90\%). In comparison with anthropometric data of facial proportions, patients with CFNS had a significantly different face in multiple respects. An overview of all phenotypic features is shown. Patients with EFNB1 mutations have a clear phenotype. This study will facilitate genetic counseling of parents and patients, and contribute to the diagnostic and screening process of patients with suspected CFNS.

European Journal of Human Genetics (2014) 22, 995-1001; doi:10.1038/ejhg.2013.273; published online 27 November 2013

Keywords: phenotype; craniofrontonasal; dysplasia; syndrome; facial cleft

\section{INTRODUCTION}

Craniofrontonasal syndrome (CFNS), also known as craniofrontonasal dysplasia, was identified as a specific subpopulation of frontonasal dysplasia, first delineated in a study by Cohen in 1979. ${ }^{1}$ Subsequently, many other studies have focused on the manifestation of this syndrome. Most commonly depicted phenotypic features were coronal synostosis, ${ }^{1-11}$ hypertelorism, ${ }^{1,4-14}$ bifid nasal tip, ${ }^{1,4,5,8-10,12,13}$ frizzy and curly hair ${ }^{8-11,15}$ and longitudinal ridging and splitting of nails. ${ }^{3,4,6,8,9,11-13}$ It became clear that the majority of CFNS patients were female. In addition, the female patients appeared to be affected more severely than male carriers, who showed only few mild signs or no clear features at all. A genetic basis was likely, because families with multiple affected members were reported..$^{2-4,6-9,12}$ However, there seemed to be a genetic paradox, as all daughters of affected males displayed severe signs of CFNS, but no male-to-male transmission was seen and affected males portrayed only mild or no signs. Therefore, multiple modes for inheritance were proposed; germline mosaicism, autosomal dominant with sex-influenced expression, X-linked dominant and metabolic interference. ${ }^{2-4,6-9}$

The mystery was unraveled by a combination of results of multiple studies. ${ }^{16-18}$ The disease locus was finally claimed to be within Xq13.1 and loss of function mutations in EFNB1 were proven to cause CFNS. ${ }^{10,18-30}$ EFNB1 encodes ephrin-B1, which is a transmembrane ligand for Eph receptor tyrosine kinases. Because of random $\mathrm{X}$-inactivation heterozygous females are uniquely mosaic and by consequence a cell either does or does not produce a functional protein. These proteins are important for cell-cell contact, migration and pattern formation in the developmental process of the embryo. ${ }^{31}$ The random pattern of expressing and non-expressing patches therefore leads to an abnormal sorting in cells, and in addition to ectopic tissue boundaries between these zones. The term for this process is called 'cellular interference. ${ }^{10}$ In hemizygous males, all cells are unable to produce a functional protein, and therefore this phenomenon cannot occur. Normal boundaries are probably maintained through an alternative mechanism, ${ }^{25}$ which could be via an ephrin redundancy ${ }^{25}$ and promiscuity of the ephrin ligand/ receptor system. ${ }^{10}$ An explanation for the few severely affected males reported in literature ${ }^{6-8,32}$ could be a mosaicism in these patients, in which the wild type to mutant ratio should be similar to that in heterozygous CFNS females. ${ }^{23,26,33}$ Additional mechanisms were recently added to the phenotypic manifestation. Not only cellular interference, but also an impaired signaling capacity of ephrin-B1 and improper regulation of gap junctional communication could be responsible for the pathogenic process in CFNS expression. ${ }^{24,28}$ A genotype-phenotype correlation has not been proven, and previous studies suggest that this is unlikely. ${ }^{26}$

Taken together, around $20 \%$ of the patients screened for CFNS did not display a mutation in the EFNB1 gene. ${ }^{21,23,25}$ Multiple explanations have been proposed, one of which is misdiagnosis of some of the included patients. ${ }^{25}$ Studies following the discovery of the causal gene predominantly describe the location of new mutations, combined with a brief outline of phenotypic features of small families

${ }^{1}$ Department of Plastic and Reconstructive Surgery, Erasmus MC, University Medical Center, Rotterdam, The Netherlands; ${ }^{2}$ Weatherall Institute of Molecular Medicine, University of Oxford, John Radcliffe Hospital, Oxford, UK; ${ }^{3}$ Department of Clinical Genetics, Erasmus MC, University Medical Center, Rotterdam, The Netherlands

${ }^{*}$ Correspondence: Dr MEP van den Elzen, Department of Plastic and Reconstructive Surgery, Erasmus MC University Medical Center, Room Ee 15.91, Dr Molewaterplein 50, Rotterdam 3015 GE, The Netherlands. Tel: +31 10 7043291, Fax: +31 10 7044685; E-mail: marijke.vandenelzen@gmail.com

Received 5 June 2013; revised 15 October 2013; accepted 24 October 2013; published online 27 November 2013 
or cohorts. ${ }^{18-20,22,27,29,30}$ Detailed overviews of phenotypic features of large cohorts of possible CFNS patients do exist, ${ }^{3,4,6-10,12,30}$ but it is not clear what proportion of these patients carry an EFNB1 mutation. The reports in the older literature could therefore cause confusion by reporting patients who might be improperly classified as CFNS patients.

As it is of major importance to adequately counsel patients with EFNB1 mutations and/or their parents and to improve diagnosis of new patients, more information about the phenotypic features of genuine CFNS patients with an EFNB1 mutation is needed.

\section{METHODS}

\section{Study population}

This study was conducted at the Craniofacial Unit of the Department of Plastic and Reconstructive Surgery of the Erasmus MC, University Medical Center in Rotterdam, The Netherlands. All patients with a diagnosis of CFNS based on a confirmed EFNB1 mutation who were currently under treatment, or who were treated in the past, were included in this study. A total of 23 patients (21 female, two male) were selected. Seven of these patients have been described in prior clinical studies, and the mutations of 13 patients have been published before. ${ }^{6,32,33}$

\section{Design and procedure}

A cross-sectional observational study was designed and conducted. Ethical approval was received from the board of the Medical Ethical Committee of the Erasmus MC, University Medical Center Rotterdam (MEC-2006-121).

Complete series of standardized photographs of all patients were collected, combined with a review of the patient's medical file and physical examinations. If available, radiological images were also consulted. However, as this was a retrospective study, not all images were still available and moreover could not always be used as a source of quantitative data. Patients were asked to participate for an extra physical examination and additional photographs to capture all bodily features. Some short questions on functioning and limitations of their body were asked as well. Patients or parents provided written consent for the use of patient images.

\section{Genotype-phenotype correlation}

The EFNB1 gene was examined by DNA sequence analysis of all coding exons and exon/intron boundaries. Rearrangements of EFNB1 (deletions or insertions) were sought by multiplex ligation-dependent probe analysis. For each patient, we classified the type of nucleotide change (point mutation, deletion or duplication) and the type of protein change (missense, nonsense or frameshift mutation). We also indicated in which exons (exons 1-5) the mutations were located and in which regions of the protein the mutations were located (signal peptide, receptor-binding domain, ephrin (extracellular domain), transmembrane domain, cytoplasmic domain or PDZ domain).

\section{Measurement of facial proportions}

For calculation of facial proportions, standardized (frontal and profile) photographs of all patients were printed. Selected photographs had to be taken before major surgical interventions, so genuine dimension could be evaluated. As all evaluated facial proportions were ratios, no scaling or calibration problems existed. Calculated indexes were compared with values derived from anthropometric studies. ${ }^{34}$ As illustrated in Figure 1, chosen indexes were: Intercanthal Index (Intercanthal width/ Biocular width); Upper Face Index (upper face height/face width); Nasal Protrusion-Nose Height Index (nasal tip protrusion/nose height); Nose-Craniofacial Height Index (nose height/craniofacial height); Nose-Upper Face Height Index (nose height/ upper face height) and Upper Lip-Upper Face Height Index (upper lip height/ upper face height). Nomenclature of the mentioned indexes and measurements are directly derived from the referred anthropometric studies. ${ }^{34}$

\section{Statistical analyses}

As a measure of central tendency, percentages were calculated for categorical variables. For metric variables, the mean was used as measure of central tendency, and the standard deviation was used as a measure of dispersion. For statistical analysis, we used the Statistical Package for the Social Sciences (SPSS) for Windows, version 18.0 (Erasmus MC University Medical Center, Rotterdam, The Netherlands).

\section{RESULTS}

Twenty-three patients with classical features of CFNS and proven EFNB1 mutations (Table 1, Variants reported in this study are submitted to the LOVD database http://databases.lovd.nl/shared/ genes/EFNB1) were included in this study. The single patient excluded was a female originally suspected to have CFNS, but who had no identified EFNB1 mutation; in retrospect (with the findings of this study) she would clinically not be classified again as having CFNS. A total of 21 female and 2 male CFNS patients were identified. Five patients refused to participate for the additional physical examination and photographs. The main reason for not participating was an emotional or psychological problem with their bodily features, and seeing 'no use' in participating. One patient could not be contacted. Patients who did not want to participate, however, had had standardized photographs taken during their treatment at least of their face, and sometimes hands, feet and chest. Medical files were available for all selected patients. As a result, the denominator of some of the observed features was lower than the total of 23 patients. Other features (eg, breast anomalies) could not be scored in all patients, because these individuals were either male or too young to have developed breasts. In addition, one of our patients was a baby aged 6 months, so many features could not be scored. In these cases of incomplete data, fractions are given instead of percentages.

Five patients were heterozygous for the familial mutation identified in the index patient of the family. Regarding the intrafamilial variability, the following most obvious features were found: concerning the patient who was a daughter of a carrier male with a very mild phenotype, no comparison could be made because the daughter obviously showed a very different phenotype. Concerning the affected mother and daughter pair, both patients were equally affected in facial and bodily features. The daughter, however, was born with several cardiac anomalies, dextroposition of the heart, two superior venae cavae, a bidirectional shunt and an atrial septal defect. The two affected sisters differed slightly in facial features, as one of the sisters had more pronounced epicanthic folds, whereas the other had a more obvious orbital dystopia. In addition, one of them had a coloboma of the iris, a strabismus sursoadductorius, and a difference in length of legs (asymmetrical lower limb shortness).

Overall, we scored 107 phenotypic variables in the cohort of 23 patients. We also checked the genotype of our patients and used four variables to describe the genotype (type of nucleotide change, type of protein change, involved exon and region of protein affected). Many variables consisted of multiple possible values, leading to a large variation of both genotype and phenotype, and no genotypephenotype correlation could be detected.

\section{General features}

The average age of patients at evaluation was 18.0 years (range $0.5-44$ years) with 12 adults (age 16 years and above). Analysis of the body mass index revealed that $28 \%(n=5)$ were underweight and $11 \%$ $(n=2)$ were overweight. The span-length-ratio had a mean of 0.93 (range 0.77-0.99), indicating that in most patients their arm span was 


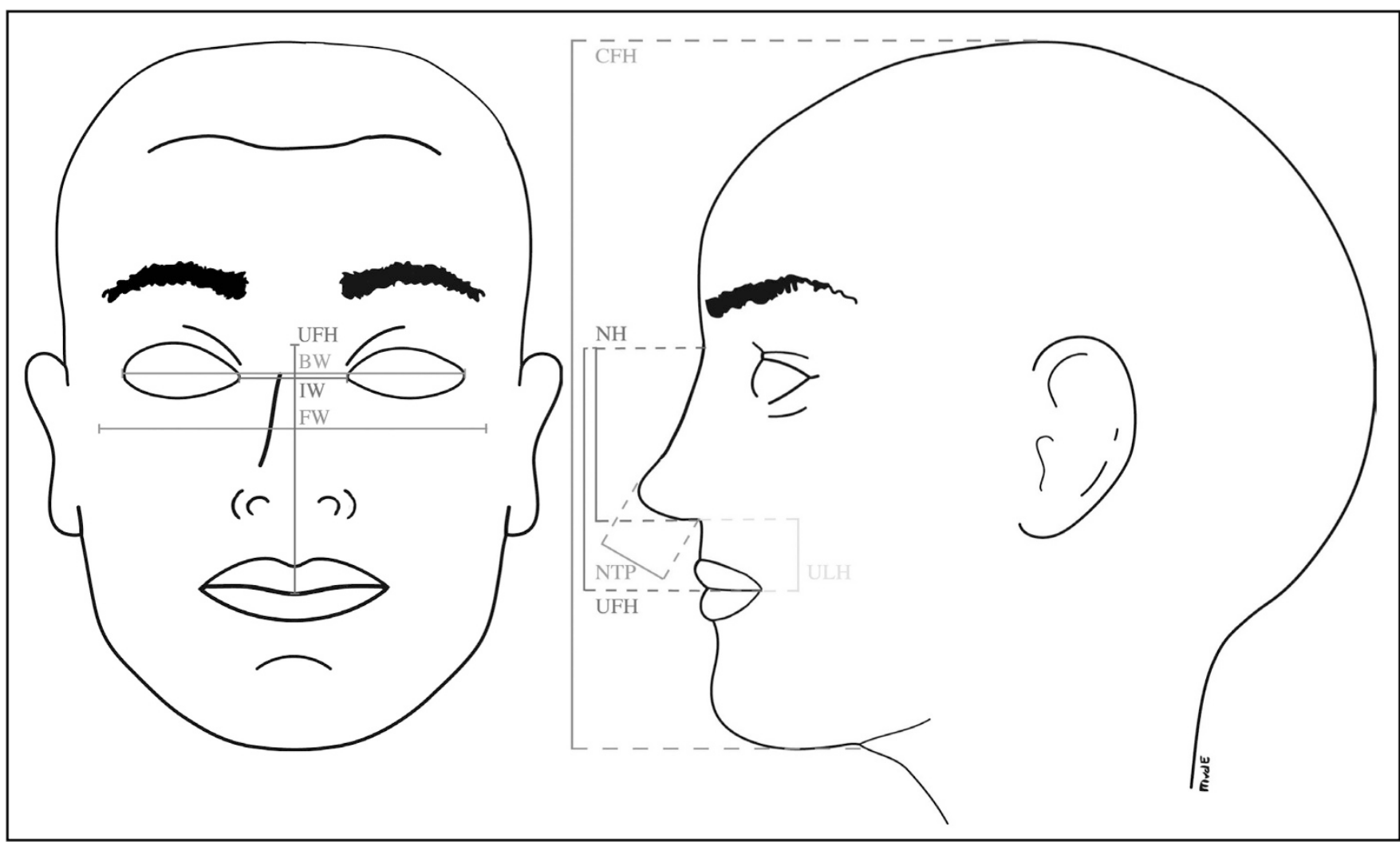

Figure 1 Anthropometric facial proportions. BW, biocular width; CFH, craniofacial height; FW, face width; IW, intercanthal width; NH, nose height; NTP, nasal tip protrusion; UFH, upper face height; ULH, upper lip height.

Table 1 Overview of mutations in the EFNB1 gene ${ }^{a}$

\begin{tabular}{|c|c|c|c|c|c|}
\hline Gender & Nucleotide change & Protein change & Exon & Protein region & Inheritance \\
\hline 1 Female $^{33}$ & c. $[=/-95 \mathrm{~T}>\mathrm{C}]$ & p.? & 5'UTR & NA & 2 \\
\hline 2 Male $^{32,33}$ & c. $\left[=/ 412-399 \_1038+7004 \mathrm{del}\right]$ & p.? & $3-5$ & Ephrin partially, TM and CY & 3 \\
\hline 3Female & c. $1 \mathrm{~A}>\mathrm{G}$ (mosaic) & p.0? & 1 & $\mathrm{SP}$ & 3 \\
\hline 4 Female 23 & c. $30 \mathrm{C}>\mathrm{T}$ & p.(Lys11fs) $)^{b}$ & 1 & SP & 1 \\
\hline 5 Female ${ }^{6,23}$ & c. $109 \mathrm{~T}>\mathrm{G}$ & p.(Trp37Gly) & 1 & RBD & 1 \\
\hline 6Female & c. $161 \mathrm{C}>\mathrm{T}$ & p.(Pro54Leu) & 2 & RBD & 0 \\
\hline 7Female & c.196delC & p.(Arg66fs) & 2 & RBD & 2 (daughter) \\
\hline 8Female & c. $228 \mathrm{C}>\mathrm{G}$ & p. $($ Tyr76*) & 2 & $\mathrm{RBD}$ & 1 \\
\hline 9Female 23 & c. $233 \mathrm{~T}>\mathrm{C}$ & p.(Leu78Pro) & 2 & RBD & 2 (mother) \\
\hline 10Female 23 & c. $233 \mathrm{~T}>\mathrm{C}$ & p.(Leu78Pro) & 2 & RBD & 2 (daughter) \\
\hline 11Female & c. $266 \mathrm{G}>\mathrm{A}$ & p.(Cys89Tyr) & 2 & $\mathrm{RBD}$ & 1 \\
\hline 12Female & c.324dupA & p.(Arg109fs) & 2 & RBD & 1 \\
\hline 13 Female $e^{6,23}$ & c. $339 \mathrm{G}>\mathrm{C}$ & p.(Lys113Asn) & 2 & RBD & 1 \\
\hline 14Female & c. $360 \mathrm{C}>\mathrm{A}$ & p.(Asn120Lys) & 2 & $\mathrm{RBD}$ & 0 \\
\hline 15 Female $^{6}$ & c. $368 \mathrm{G}>\mathrm{A}$ & p.(Gly123Asp) & 2 & RBD & 1 \\
\hline 16 Female $^{23}$ & c. $407 \mathrm{C}>\mathrm{T}$ & p.(Ser136Leu) & 3 & RBD & 0 \\
\hline 17Female & c. $451 \mathrm{G}>\mathrm{A}$ & p.(Gly151Ser) & 3 & $\mathrm{RBD}$ & 0 \\
\hline 18Female & c. $492 \_499+2$ del & p.(Gly165fs) & 3 & Ephrin & 1 \\
\hline 19 Male ${ }^{6,33}$ & c. $496 \mathrm{C}>\mathrm{T}$ (mosaic) & p. $\left(G \ln 166^{*}\right)$ & 3 & Ephrin & 3 \\
\hline $20 F_{e m a l e}^{6,23}$ & c. $496 \mathrm{C}>\mathrm{T}$ & p. $\left(G \ln 166^{*}\right)$ & 3 & Ephrin & 2 (Sister) \\
\hline 21 Female $^{6,23}$ & c. $496 \mathrm{C}>\mathrm{T}$ & p. $\left(G \ln 166^{*}\right)$ & 3 & Ephrin & 2 (Sister) \\
\hline 22Female & c.543delC & p.(Ser182fs) & 4 & Ephrin & 1 \\
\hline 23Female 23 & c.564dupT & p.(Val189fs) & 4 & Ephrin & 1 \\
\hline
\end{tabular}

Abbreviations: CY, cytoplasmic domain; Ephrin, ephrin extracellular domain; NA, not applicable; RBD, receptor-binding domain (located in the ephrin extracellular domain); SP, signal peptide; TM, transmembrane domain; UTR, untranslated region; 0 , de novo; 1 , sporadic; 2 , familial; 3 , mosaic.

Variants submitted to http://databases.lovd.nl/shared/genes/EFNB1.

aReference sequence: NG_008887.1.

bRNA analysis showed that this synonymous substitution creates a cryptic donor splice site. ${ }^{23}$ 
not equal to their total height, which is in contrast to the normal population.

\section{Skull and face}

As can be seen in Table 2 and Figure 2, the facial features of CFNS patients differ significantly from the normal population (more than 2 SDs above the mean). ${ }^{34}$ As expected, the intercanthal distance is much greater. The upper face (base of the nose to height of the commissure of the mouth) is relatively small compared with the width of the face (lateral points of zygoma). Compared with the height of the nose, the protrusion of the tip is relatively high in patients. However, the height of the nose itself is significantly shorter, compared with both the total face as well as the upper face. In contrast, the upper lip is larger than normal.

Craniosynostosis was seen in $78 \%$ of all patients; $22 \%(n=5)$ had a left-sided coronal synostosis, $4 \%(n=1)$ had a right-sided coronal synostosis, $48 \%(n=11)$ had a bilateral synostosis of the coronal suture and $4 \%(n=1)$ had a bilateral coronal synostosis with synostosis of the sagittal suture. One patient had her craniosynostosis corrected abroad before her first presentation, and the exact type of synostosis was unclear. All patients with craniosynostosis needed a surgical correction. A very large anterior fontanelle with delayed closure was present in $6 / 18$. Two patients had agenesis of the corpus callosum agenesis, with partial agenesis in another three patients. Facial asymmetry was seen in $19 / 22$ of all patients, with a degree ranging from mild to severe; three of these patients had no history of craniosynostosis. A diminished development of the maxilla was sometimes observed (4/22), at different ages and of variable degree. In addition, one patient had a groove in the middle of her alveolar ridge.

\section{Hair}

In our population, $65 \%(n=15)$ of all patients had a widow's peak, and $26 \%(n=6)$ had a low anterior hairline. The hair itself was dry and with frizzy curls in $12 / 22$, dry and with loose curls in $8 / 22$, and $2 / 22$ showed normal hair. Parents reported that hair usually changed around 6-12 months, from soft baby hair into dry curly hair.

\section{Zone of the orbits and eyes}

All patients, $100 \%(n=23)$, displayed hypertelorism, with a variable degree from mild to severe. At the time of evaluation, six patients were still too young to undergo a correction of their hypertelorism, but will probably be operated on in the future. Two adults with a relatively mild form did not require surgical intervention, the other 15 patients underwent a surgical correction. Orbital dystopia was seen in $10 / 22$ of them, five of the patients had no history of craniosynostosis, in seven of them the orbital dystopia was evident before any surgical intervention. Downslanting of the palpebral fissures was present in $35 \%(n=8)$, with variable severity, whereas an upslant of the palpebral fissures was present in $48 \%(n=11)$, although usually only mild. Epicanthic folds were frequent, and were unilateral in $39 \%(n=9)$ cases and bilateral in 39\% $(n=9)$ cases. An interrupted hairline of the eyebrow was seen in $70 \%(n=16)$ of patients. Rare observations were a coloboma of the iris $(n=1)$ and heterochromia of the iris $(n=1)$.

Table 2 Difference of facial proportions of CFNS patients compared with anthropometric means ${ }^{34}$ (expressed in average standard deviations from mean)Abbreviation: CFNS, craniofrontonasal syndrome

\begin{tabular}{lccccc}
\hline & $\begin{array}{c}\text { Intercanthal } \\
\text { Index }\end{array}$ & $\begin{array}{c}\text { Upper Face } \\
\text { Index }\end{array}$ & $\begin{array}{c}\text { Protrusion-Nose Height } \\
\text { Index }\end{array}$ & $\begin{array}{c}\text { Nose-Craniofacial Height } \\
\text { Index }\end{array}$ & $\begin{array}{c}\text { Nose-Upper Face Height } \\
\text { Index }\end{array}$ \\
\hline Patients <6 years & $+5.3 \mathrm{SD}$ & $-2.1 \mathrm{SD}$ & $+1.7 \mathrm{SD}^{\mathrm{a}}$ & $-3.4 \mathrm{SD}^{\mathrm{b}}$ & $-4 \mathrm{SD}^{\mathrm{b}}$ \\
Patients $>6$ years & $+4.7 \mathrm{SD}$ & $-1.5 \mathrm{SD}$ & $+1.6 \mathrm{SD}$ & $-1.6 \mathrm{SD}$ & $-2.0 \mathrm{SD}$ \\
Total group & $+5.0 \mathrm{SD}$ & $-1.8 \mathrm{SD}$ & $+1.7 \mathrm{SD}$ & $-2.5 \mathrm{SD}$ & $-3.0 \mathrm{SD}$ \\
\hline
\end{tabular}

aYoungest age of reference group is 6 years, difference probably slightly bigger if adequate reference would be available.

byoungest age of reference group is 6 years, difference probably slightly smaller if adequate reference would be available.

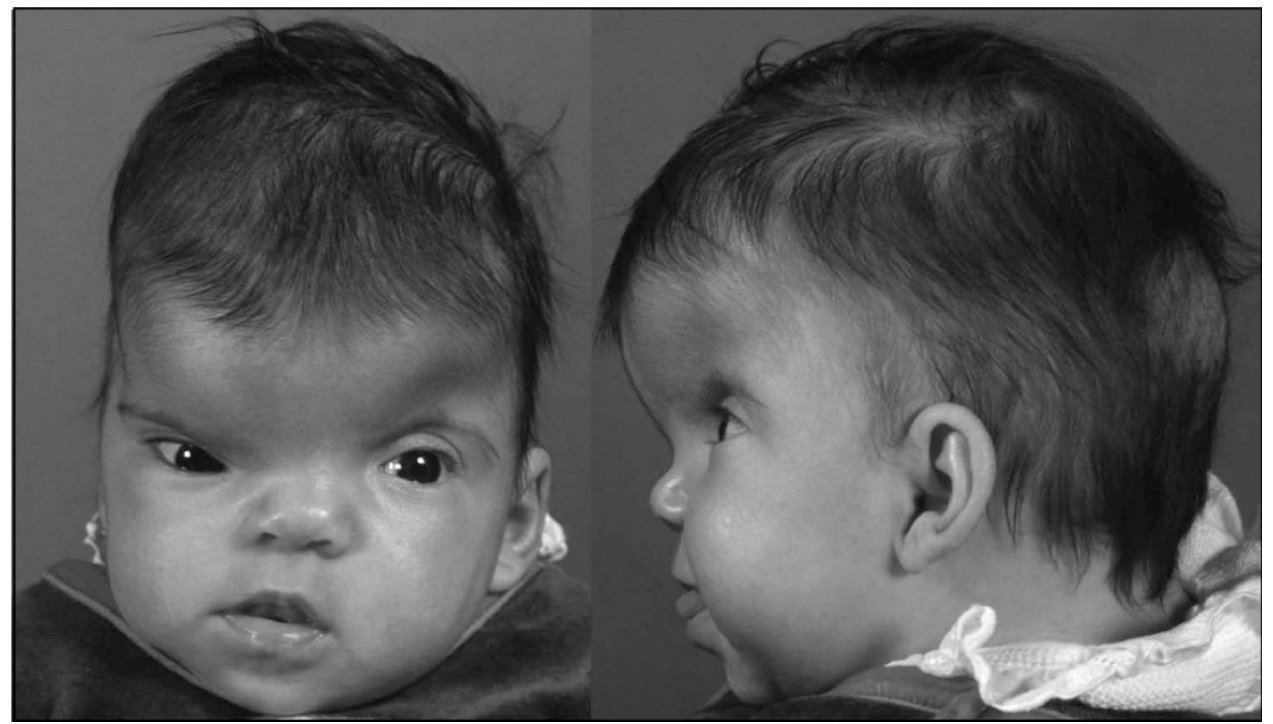

Figure 2 Patient displaying typical aberrant facial proportions. 


\section{Ocular function}

Before a correction of the hypertelorism or orbital dystopia, a substantial number of ophthalmologic abnormalities were observed. The most common anomaly was strabismus (9/22), subdivided into divergent $(n=3)$, sursoadductorius $(n=4)$ and convergent $(n=2)$ types, sometimes in combination with a dissociated vertical deviation $(n=5)$. Nystagmus was also a common finding (9/22 patients): four had congenital nystagmus and four had latent nystagmus. Hypermetropia was present in three, two of them had a very high astigmatism and one had solitary high stigmatism. Amblyopia was identified in two and an absent oblique superior muscle was found in one patient.

Ears

Low set ears were a common finding, $52 \%(n=12)$, whereas only two patients had an abnormal shape of the external ear.

\section{Zone of the nose}

Nearly, all patients $(91 \%, n=21)$ displayed a bifid tip of the nose. The same can be said for an indentation in the columella, 91\% $(n=21)$, although not all patients with a bifid tip also had this indentation. A broad nasal base, 70\% $(n=16)$, and flat nasal bridge, $43 \%(n=10)$, were frequently observed. One patient had a fistula in the dorsum of her nose with an intracranial connection.

\section{Zone of the mouth, maxilla and mandible}

A common observation was a tent-shaped mouth in 39\% $(n=9)$, and a mild keel-shaped maxilla in $35 \%(n=8)$. Crowding of the teeth was seen in $23 \%(n=5)$. Hypoplasia of the maxilla was reported in $4 / 22$ patients, whereas $2 / 22$ had a mandibular prognathism. A unilateral right-sided cleft lip and palate was seen in only one patient, whereas one other patient had a very mild notch in the midline of her upper lip.

\section{Zone of the neck, shoulders, chest and back}

A true short and webbed neck was present in 12/18 patients and in addition a mild webbing or pseudo-webbing of the neck was present in the other 6/18 patients. Rounded and sloping shoulders, often rather narrow, were observed in 16/18. Sprengel's deformity of the shoulders (defined as one shoulder blade that sits higher on the back than the other) was quite common as well (8/12). Three patients displayed an axillary pterygium; unilateral in two patients, bilateral in one. A low implantation of the breasts was seen in the majority of patients (19/21), and in addition most of them had asymmetrical heights of their nipples (11/19). Patients who were in their adolescence or adulthood also displayed an asymmetry of the breast volume (6/8). Looking at the chest wall itself, revealed a pectus excavatum in $11 / 17$ patients, although mild in most cases. Four patients were affected with both breast asymmetry and a pectus excavatum. All of the above are illustrated in Figure 3. Scoliosis was diagnosed in 6/13 patients, but none of the patients needed surgery.

\section{Upper extremity}

All patients $(100 \%, n=23)$ had a longitudinal ridging and/or splitting of nails toward the end, although the number of digits affected and severity differed. Only two patients were born with an extra digit (9\%), and only three had a complete or incomplete syndactyly (13\%). A clinodactyly of one or more digits was frequent $(74 \%, n=17)$. A restricted range of motion of the arms, affecting either abduction or elevation above the head, was present in the majority of patients $(15 / 17)$. This is probably due to the aberrant position of the clavicles, in combination with the earlier mentioned Sprengel's deformity. The available radiological images of the chest revealed that patients

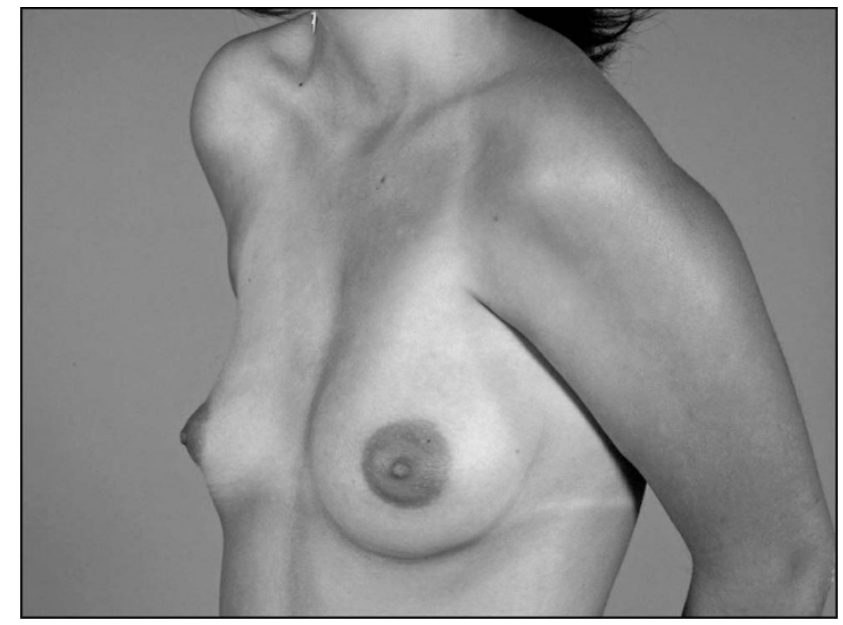

Figure 3 Patient with typical chest and breast deformities.

had either an aberrant curvature of their clavicles and/or the angle of the clavicles with the sternum was bigger than normal. Either way, this resulted in a typical higher placement of the shoulders.

\section{Lower extremity}

As was observed in the hand, all patients (20/20) had a longitudinal ridging and/or splitting of toenails toward the end, whereas both the severity as well as the number of toes affected differed. Duplication of toes was seen in $4 / 20$ patients and syndactyly in $7 / 20$ patients. Clinodactyly of at least one toe, was again a consistent finding (20/20), as illustrated in Figure 4. One patient had asymmetrical lower-limb shortness.

\section{Cardiac abnormalities}

Three patients $(13 \%)$ had problems affecting their heart. One had a patent ductus arteriosus, one had an atrial flutter of unknown origin immediately after birth and one had multiple cardiac problems: dextroposition of the heart, two superior venae cavae, a bidirectional shunt and an atrial septal defect.

\section{Miscellaneous findings}

Two patients had psoriasis and another single patient had an umbilical hernia, one male presented with cryptorchidism, another patent had a cafe-au-lait spot, one patient presented herself with an hemangioma and one patient had an episode with toddler's hypoglycemia.

\section{DISCUSSION}

Genuine CFNS patients, with proven EFNB1 mutations, have a clear and very distinguishable phenotype. However, a detailed overview of the phenotypic features of a large cohort of proven CFNS patients has not previously been presented in the literature. As expected, some of the previous reports can cause confusion by reporting patients who were possibly incorrectly diagnosed with CFNS. This probably explains the majority of the $\sim 20 \%$ of apparent CFNS patients without an EFNB1 mutation. ${ }^{21,23,25}$ The results from this study make the diagnosis of some patients presented in literature therefore doubtful, based on their different facial proportions and dissimilar phenotype. ${ }^{7,14,30}$ In other studies, some patients were classified as having frontonasal dysplasia, whereas they actually match the typical phenotype of CFNS. ${ }^{13,32,35}$ 


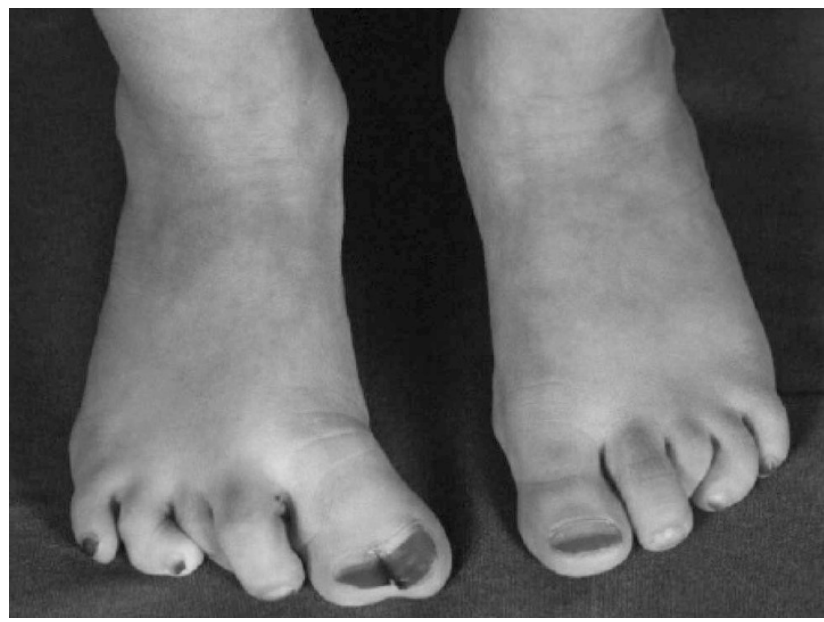

Figure 4 Patient displaying typical foot and toe deformities.

We included only three families with more than one affected member in this study. One of these comprised a father (a male carrier) and a daughter and therefore we could not draw any conclusions regarding intrafamilial variability. In the other two families, the clinical features differed highly, suggesting that intrafamilial variability can be significant. A genotype-phenotype correlation seems to be unlikely, based on this intrafamilial variability and a previous study, ${ }^{26}$ and was not found in this study.

This study leads us to the conclusion that in CFNS patients with an EFNB1 mutation, consistent features exist: hypertelorism, a certain degree of longitudinal ridging and/or splitting of nails of at least one digit or toe, a certain degree of webbed neck and a clinodactyly of one or more toes. In addition, abnormal facial proportions were observed in all patients. ${ }^{34}$ These proportions are reflected in a relatively small upper face compared with the width of the face, a very short nose, with a relatively high protrusion compared with its length and a relatively long upper lip, compared with the upper face. It must be stressed that the projection of the nose itself in comparison to the whole face is very small, but as the length is about the same as the projection, this ratio is relatively high.

Features that were present in most, but not all patients, can be regarded as 'very suggestive' of the diagnosis. These are: bifid tip of the nose $(91 \%)$, indentation of the columella (91\%), low implant of breasts (90\%), rounded, sloping and often rather narrow shoulders (89\%) with reduced range of motion of the shoulders $(88 \%)$, facial asymmetry (86\%), craniosynostosis (78\%), clinodactyly of at least one digit (74\%), aberrant form of eyebrow (70\%) and broad nasal bridge $(70 \%)$. Although similar features have been noted in previously published studies, ${ }^{4,6,8,9,11,12}$ it is not possible to compare frequency data directly because of differences in methodology.

Measurements of facial proportions are seldom reported, which is unfortunate. One study gave a description of the cranio-orbitozygomatic region, based on CT-scans compared with an age-matched control value. Besides the obvious increased interorbital distance, they also found a degree of horizontal midface retrusion demonstrated by a shortened zygomatic arch length and an expanded interzygomatic buttress distance, suggestive for a brachycephalic morphology. ${ }^{13}$ In addition, another study also described a short upper facial height ${ }^{8}$ and compared it to anthropometric measurements. However, the short upper facial height seemed to be present in only $66 \%$ of their cases. A further study mentions midface hypoplasia, ${ }^{3}$ however, they do not support it with objective data or compare it to a normal reference group. These findings are in accordance to our data.

Hopefully, this study will facilitate the diagnosis of CFNS. Based on this study, new patients should be evaluated for facial proportions and hypertelorism, they should be checked for longitudinal ridging and/or splitting of nails of at least one digit or toe, for webbed neck and for a clinodactyly of one or more toes. Furthermore, other bodily features should be compared with the ones given in this paper.

It is likely that not all possible phenotypic features of CFNS are present within our population. A few other features that were not evaluated in this study are presented in the literature: myoclonus, poor hearing, pelvic kidney, bilateral vesico-ureteral reflux, hip girdle anomalies, ${ }^{11}$ median cleft lip/palate, ${ }^{8}$ asymmetric mandible. ${ }^{9}$ Additional features that have been reported in other studies of patients with EFNB1 mutations include: diaphragmatic hernia, ${ }^{18,19,21,27}$ dysplastic clavicles and clavicle pseudoarthrosis, ${ }^{8-12,18,20,23}$ accessory nipples, ${ }^{10}$ high arched palate, ${ }^{4,9-12,32}$ uterus arcuatus, ${ }^{10}$ duplication of uterus, kidneys and ureters, ${ }^{10}$ and low posterior hairline. ${ }^{8,12,22}$ Furthermore, some studies state that CFNS patients have a normal intelligence, $8,10,12,15$ whereas others claim that some may have learning difficulties to a variable degree. , $^{3,10,23,32}$ In this study, intelligence was not measured.

One of the limitations of this study is the low number of males included. Although CFNS manifests particularly in females, and affected males express significantly less features, a clear phenotype of hemizygous males could not be given, as both of the two males evaluated carried mosaic mutations, and as a consequence were as severely affected as the females. ${ }^{32}$ On the other hand, we believe that the number of clinically evaluated CFNS patients with proven EFNB1 mutations in this study is unique in its size, and therefore adds value to the current literature. Future research could focus on the psychological and intellectual development and surgical impact.

\section{CONFLICT OF INTEREST}

The authors declare no conflict of interest.

\section{ACKNOWLEDGEMENTS}

We thank Professor Dr Han Brunner for analyzing the samples of multiple patients. This study was funded by the CZ Fonds and Stichting Achmea Gezondheidszorg (Dutch Health Insurance Companies) and by the Wellcome Trust (093329 to SRFT and AOMW).

1 Cohen MM Jr: Craniofrontonasal dysplasia. Birth Defects Orig Artic Ser 1979; 15 85-89.

2 Reynolds JF, Haas RJ, Edgerton MT, Kelly TE: Craniofrontonasal dysplasia in a three-generation kindred. J Craniofac Genet Dev Biol 1982; 2: 233-238.

3 Grutzner E, Gorlin RJ: Craniofrontonasal dysplasia: phenotypic expression in females and males and genetic considerations. Oral Surg Oral Med Oral Pathol 1988; 65: 436-444.

4 Kere J, Ritvanen A, Marttinen E, Kaitila I: Craniofrontonasal dysostosis: variable expression in a three-generation family. Clin Genet 1990; 38: 441-446.

5 Suzuki H, Nara T, Minato S, Kamiishi H: Experience of surgical treatment for craniofrontonasal dysplasia. Tohoku J Exp Med 1991; 164: 251-257.

6 Kapusta L, Brunner HG, Hamel BC: Craniofrontonasal dysplasia. Eur J Pediatr 1992; 151: 837-841.

7 Natarajan U, Baraitser M, Nicolaides K, Gosden C: Craniofrontonasal dysplasia in two male sibs. Clin Dysmorphol 1993; 2: 360-364.

8 Saavedra D, Richieri-Costa A, Guion-Almeida ML, Cohen MM Jr: Craniofrontonasal syndrome: study of 41 patients. Am J Med Genet 1996; 61: 147-151.

9 Orr DJ, Slaney S, Ashworth GJ, Poole MD: Craniofrontonasal dysplasia. Br J Plast Surg 1997; 50: 153-161.

10 Wieacker $\mathrm{P}$, Wieland I: Clinical and genetic aspects of craniofrontonasal syndrome: towards resolving a genetic paradox. Mol Genet Metab 2005; 86: 110-116.

11 Kawamoto HK, Heller JB, Heller MM et al: Craniofrontonasal dysplasia: a surgical treatment algorithm. Plast Reconstr Surg 2007; 120: 1943-1956.

12 Young ID: Craniofrontonasal dysplasia. J Med Genet 1987; 24: 193-196. 
13 Moffat SM, Posnick JC, Pron GE, Armstrong DC: Frontonasal and craniofrontonasal dysplasia: preoperative quantitative description of the cranio-orbito-zygomatic region based on computed and conventional tomography. Cleft Palate Craniofac J 1994; 31. 97-105.

14 Mahore A, Shah A, Nadkarni T, Goel A: Craniofrontonasal dysplasia associated with Chiari malformation. J Neurosurg Pediatr 2010; 5: 375-379.

15 Hurst J, Baraitser M: Craniofrontonasal dysplasia. J Med Genet 1988; 25 $133-134$.

16 Wieland I, Jakubiczka S, Muschke P et al: Mapping of a further locus for X-linked craniofrontonasal syndrome. Cytogenet Genome Res 2002; 99: 285-288.

17 Compagni A, Logan M, Klein R, Adams RH: Control of skeletal patterning by ephrinB1EphB interactions. Dev Cell 2003; 5: 217-230.

18 Twigg SR, Kan R, Babbs C et al: Mutations of ephrin-B1 (EFNB1), a marker of tissue boundary formation, cause craniofrontonasal syndrome. Proc Natl Acad Sci USA 2004; 101: 8652-8657.

19 Wieland I, Jakubiczka S, Muschke P et al: Mutations of the ephrin-B1 gene cause craniofrontonasal syndrome. Am J Hum Genet 2004; 74: 1209-1215.

20 Shotelersuk V, Siriwan P, Ausavarat S: A novel mutation in EFNB1, probably with a dominant negative effect, underlying craniofrontonasal syndrome. Cleft Palate Craniofac J 2006; 43: 152-154.

21 Wieland I, Reardon W, Jakubiczka S et al: Twenty-six novel EFNB1 mutations in familial and sporadic craniofrontonasal syndrome (CFNS). Hum Mutat 2005; 26: $113-118$.

22 Vasudevan PC, Twigg SR, Mulliken JB, Cook JA, Quarrell OW, Wilkie AO: Expanding the phenotype of craniofrontonasal syndrome: two unrelated boys with EFNB1 mutations and congenital diaphragmatic hernia. Eur J Hum Genet 2006; 14: 884-887.

23 Twigg SR, Matsumoto K, Kidd AM et al: The origin of EFNB1 mutations in craniofrontonasal syndrome: frequent somatic mosaicism and explanation of the paucity of carrier males. Am J Hum Genet 2006; 78: 999-1010.
24 Davy A, Bush JO, Soriano P: Inhibition of gap junction communication at ectopic Eph/ephrin boundaries underlies craniofrontonasal syndrome. PLoS Biol 2006; 4: e315.

25 Wallis D, Lacbawan F, Jain M et al: Additional EFNB1 mutations in craniofrontonasal syndrome. Am J Med Genet A 2008; 146A: 2008-2012.

26 Wieland I, Makarov R, Reardon W et al: Dissecting the molecular mechanisms in craniofrontonasal syndrome: differential mRNA expression of mutant EFNB1 and the cellular mosaic. Eur J Hum Genet 2008; 16: 184-191.

27 Hogue J, Shankar S, Perry H, Patel R, Vargervik K, Slavotinek A: A novel EFNB1 mutation (c.712delG) in a family with craniofrontonasal syndrome and diaphragmatic hernia. Am J Med Genet A 2010; 152A: 2574-2577.

28 Makarov R, Steiner B, Gucev Z, Tasic V, Wieacker P, Wieland I: The impact of CFNScausing EFNB1 mutations on ephrin-B1 function. BMC Med Genet 2010; 11: 98

29 Apostolopoulou D, Stratoudakis A, Hatzaki A et al: A novel de novo mutation within EFNB1 gene in a young girl with Craniofrontonasal syndrome. Cleft Palate Craniofac $J$ 2011; 49: 109-113.

30 Zafeiriou DI, Pavlidou EL, Vargiami E: Diverse clinical and genetic aspects of craniofrontonasal syndrome. Pediatr Neurol 2011; 44: 83-87.

31 Klein R: Eph/ephrin signaling in morphogenesis, neural development and plasticity. Curr Opin Cell Biol 2004; 16: 580-589.

32 Kwee ML, Lindhout D: Frontonasal dysplasia, coronal craniosynostosis, pre- and postaxial polydactyly and split nails: a new autosomal dominant mutant with reduced penetrance and variable expression? Clin Genet 1983; 24: 200-205.

33 Twigg SR, Babbs C, van den Elzen ME et al: Cellular interference in craniofrontonasal syndrome: males mosaic for mutations in the X-linked EFNB1 gene are more severely affected than true hemizygotes. Hum Mol Genet 2013; 22: 1654-1662.

34 Farkas L, Munro I: Anthropometric Facial Proportions in Medicine, 1 edn. Springfield, Illinois: Charles C Thomas, 1987.

35 Reardon W, Temple IK, Jones B, Baraitser M: Frontonasal dysplasia or craniofrontonasal dysplasia and the Poland anomaly? Clin Genet 1990; 38: 233-236. 